\title{
Out Door Tri -Sequential (External Rotation- Adduction-Internal Rotation) Technique for Acute Traumatic Anterior Shoulder Dislocation
}

\author{
Abhishek Agarwal ${ }^{1}$, Ekansh Debuka ${ }^{2}$, Ajai Singh ${ }^{3}$, Vineet Sharma ${ }^{4}$, Bipin Kumar \\ ${ }^{1}$ Assistant Professor, Department of Orthopaedics, King Georges Medical University, Lucknow, India \\ ${ }^{2}$ Senior Resident, Department of Orthopaedics, King Georges Medical University, Lucknow, India \\ ${ }^{3}$ Professor, Department of Orthopaedics, King Georges Medical University, Lucknow, India \\ ${ }^{4}$ Professor and Head, Department of Orthopaedics, King Georges Medical University, Lucknow, India \\ 5 Junior Resident, Department of Orthopaedics, King Georges Medical University, Lucknow, India \\ *Corresponding Author: Abhishek Agarwal, Assistant Professor, Department of Orthopaedics, King Georges Medical University, \\ Lucknow, India.
}

Received: June 06, 2019; Published: June 17, 2019

DOI: 10.31080/ASOR.2019.02.0067

\begin{abstract}
Background: Shoulder dislocations accounts for half of the all joint dislocations, and of them 95-98\% are anterior dislocations. This prospective study was conducted to delineate the role of tri-sequential technique in reduction which is easy, safe and reproducible.

Materials and Methods: This Prospective study was conducted in Department of Orthopaedics, King George's Medical University, Lucknow over a period Between Jan 2017-July 2018 al shoulder were reduced either in outdoor or orthopedic emergency unit which is a tertiary care centre. Where we evaluated type of dislocations, safety, effectivity of the maneuver and comparison with the other technique was done.

Results: Out of 34 patients, $100 \%$ success rate was observed in only dislocations and average time in reduction was found to be $<2$ mins. The Average VAS pain Scores experienced while reduction was ranged $<5$. None of the patients encountered any complication. Conclusion: This Tri-sequential technique is very safe, reliable and easily reproducible even in the hands of level 1 health care provider without any risk of complication which even does not require any assistant or hospitalization.
\end{abstract}

Keywords: Tri-sequential; Anterior Shoulder Dislocation; External Rotation; Adduction; Internal Rotation

\section{Introduction}

Today's era of fast outdoor life exposes the individuals to various minor and major road traffic accidents in which dislocations represent a vast majority of repercussions after trauma. shoulder dislocation accounts for almost half them. 95 to 98 percents are anterior dislocations due to shallow concavity of glenoid cup which makes shoulder joint highly mobile and unstable [1-4]. It should not be forgotten that in countries like India these emergencies are first encountered by local practitioners (non orthopedic personnel) who don't even know the anatomy and various forces governing the shoulder joint, mechanism of injury. These paramedical staff or many at times quacks land up in failure of reduction or creating an iatrogenic fracture by attempting reduction by unaccustomed traction without knowing the mechanism. Though there are multiple techniques described traditionally but at present time no proper protocol is defined which governs pre to post reduction scenario with follow up tenure various questions are still unanswered [5,6] e.g.

- Most easy and safe method

- Requirement of intra articular analgesia

- Sedation

- General anesthesia

So in our study we are reporting our experiences of using this Tri-Sequential technique to reduce acute traumatic anterior shoulder dislocation which is easy, safe and reproducible maneuver and can give consistent results even in the hands of level I health care providers.

\section{Materials and Method}

This Prospective study of managing Acute traumatic anterior shoulder dislocation using this trisequential technique was conducted in Department of Orthopedic, King Georges Medical Univer- 
sity, Lucknow OPD and Orthopedic emergency unit between Jan 2017-July 2018 and is being continued. After getting approval of Institutional Ethics Committee, Research Cell, KGMU, Lucknow.

\section{Reduction technique}

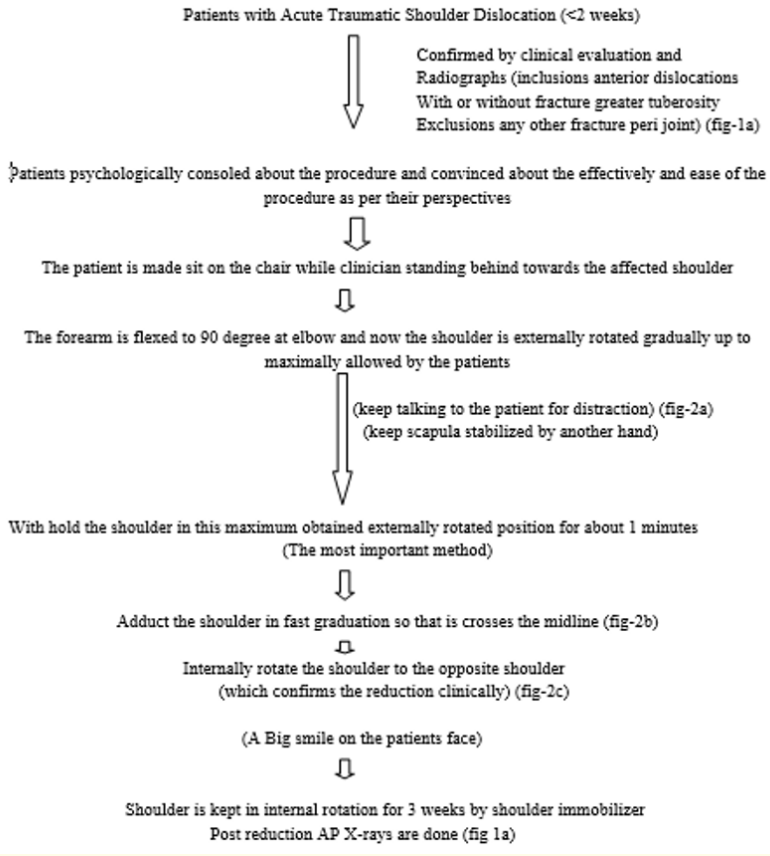

Figure

\section{Results and Discussion}

Patients were treated at time zero by clinicians (Author and team).

Out of 34 patients, $17.6 \%(6)$ reached us after a week with their shoulder manipulated elsewhere which indicates the earlier handling of their shoulder by inexperienced hands with faulty technique, in comparison to all other traditional methods of reduction our technique requires no sedation, no analgesia, no assistant and does not make patient to lie supine (table 1). In various other maneuvers like milch or kocher's [9-13,15-17] a form of traction is required which not only produce a great amount of patient discomfort and pain aggravation but makes reduction more difficult by inducing muscle spasm. This technique also forbids the use of any prop like traction towel or stockinet. Time required in our reduction technique was experienced to be less than 2 minutes (mean $1.58 \mathrm{~min}$ ). Our technique is an outdoor technique which does not need any assistant or hospitalization (Table 1).

\begin{tabular}{|c|c|c|}
\hline $\begin{array}{l}\text { Out of } 34 \text { patients } \\
\text { Male/ Female } \\
-28 / 06\end{array}$ & & \\
\hline$<30$ yrs & \multicolumn{2}{|c|}{13} \\
\hline $30-50$ yrs & \multicolumn{2}{|c|}{15} \\
\hline$>50$ yrs & \multicolumn{2}{|c|}{06 with mean age 48} \\
\hline \multirow[t]{2}{*}{25 dislocations } & \multirow{2}{*}{$\begin{array}{c}09 \text { dislocation with } \\
\text { fracture greater } \\
\text { tuberosity }\end{array}$} & 4 undisplaced \\
\hline & & 5 displaced \\
\hline \multirow[t]{3}{*}{$\begin{array}{l}\text { Average time of } \\
\text { reduction }\end{array}$} & & $\begin{array}{l}1.58 \text { minutes in } \\
\text { only dislocations }\end{array}$ \\
\hline & \multicolumn{2}{|c|}{$\begin{array}{l}2.5 \text { minutes in dislocations with fracture } \\
\text { got reduced in first attempts }\end{array}$} \\
\hline & \multicolumn{2}{|c|}{$\begin{array}{l}5.6 \text { minutes which required } 2^{\text {nd }} \text { attempts } \\
\text { including the recess period }\end{array}$} \\
\hline \multicolumn{2}{|c|}{$\begin{array}{l}\text { Pain VAS Score experienced during the } \\
\text { maneuver }\end{array}$} & No patients \\
\hline \multicolumn{2}{|l|}{$0-2$} & 0 \\
\hline \multicolumn{2}{|l|}{$2-5$} & 24 \\
\hline \multicolumn{2}{|l|}{$5-8$} & 10 \\
\hline \multicolumn{2}{|l|}{$>8$} & 0 \\
\hline
\end{tabular}

Table 1. Observed mean findings.

Average VAS score experienced by subjects while reduction is less than 5 (as is performed making patient comfortable and kept distracted to different talks while the maneuver) which is very less as compared to an average of 8 in other traditionally described ones (Table 1).

We experienced $100 \%$ success of reduction in plain dislocation or with undisplaced fracture greater tuberosity in first attempt, while $85 \%$ success rate in dislocation with displaced fracture greater tuberosity in either first or subsequent attempt. Rest 15\% required reduction under general anesthesia as with displaced fracture greater tuberosity, the misaligned rotator cuff hinders the reduction.

Osteoarthritis is a widely prevalent degenerative condition which produces considerable morbidity. Dry needling in various forms has been administered in the management of osteoarthritis. While the exact mechanism of action is hard to predict it appears dry needling has reasonable usefulness in the treatment of osteoarthritis knee.

Kuhn [7] presented the best available answer to many available methods regarding various management protocol but reports limited data available regarding the best one and premedication. Baykal (14) and colleagues reported the use of scapular manipulation with considerable success rate but requires traction and an assistant (Table 2). 


\begin{tabular}{|l|c|c|c|c|c|c|}
\hline \multicolumn{1}{|c|}{ Parameter } & Milch's & Traction/countertraction & Spaso's & Stimpson's & $\begin{array}{c}\text { Kocher's } \\
\text { Trisequential } \\
\text { (ours) }\end{array}$ \\
\hline Sedation/GA & $+/-$ & $+/-$ & - & - & $+/-$ & - \\
\hline Intraarticular injection & $+/-$ & $+/-$ & $+/-$ & $+/-$ & $+/-$ & - \\
\hline Position of the patient & supine & supine & supine & prone & supine & seated \\
\hline Average time required & $5-10$ mins & $8-10$ mins & $5-8$ mins & $10-15$ mins & $\begin{array}{c}8-10 \\
\text { mins }\end{array}$ & $<2$ mins \\
\hline Assistant required & + & + & - & - & + & - \\
\hline Any prop required & $+/-$ & $+/-$ & - & - & $+/-$ & - \\
\hline traction & + & + & $+/-$ & + gravity & + & - \\
\hline Pain (VAS)perceived if not sedated & $8-10$ & $9-10$ & $6-8$ & $6-8$ & $8-10$ & $<5$ \\
\hline Success rate in first attempt & $75-95 \%$ & $80 \%$ & $90 \%$ & $70 \%$ & $75 \%$ & $100 \%$ \\
\hline
\end{tabular}

Table 2: Comparison of different parameters in Reduction amongst various techniques with our's.

Ashton and Hassan [8] carried out a review to establish whether Kocher's or Milch's technique was better but the bottom line was its just individual preference and not supported by the evidence (Table 2).

Various methods of anaesthesia are available like General anesthesia to intrarticular lidocaine described by Pradhan et al which requires vitals monitoring or hospitalization [18].

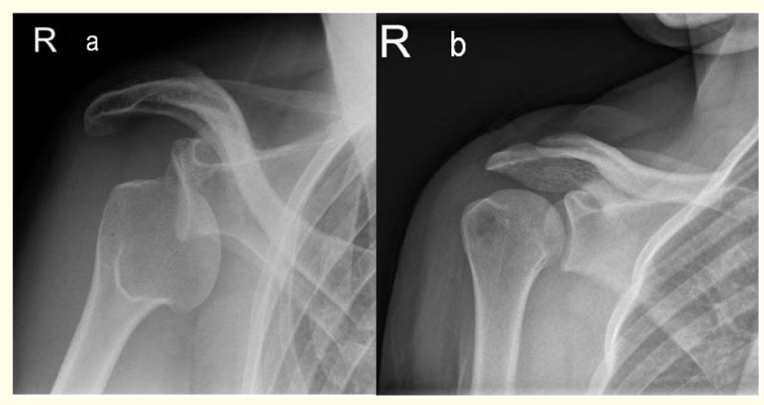

Figure 1: Showing X-ray of a. Anterior Dislocation of shoulder; b. Dislocation reduced.

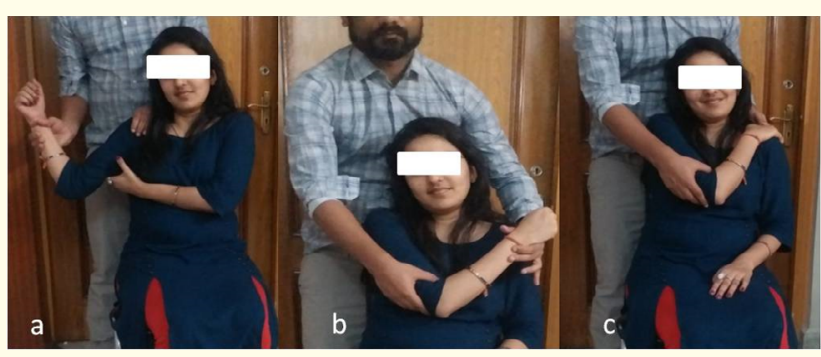

Figure 2: Showing steps of reduction maneuver; a. in maximal external rotation with scapula stablised and holding it in this position for 1 minute; $b$. Adduction in fast graduation

to mid line; c. internal rotation to opposite shoulder.

\section{Conclusion}

To conclude we can say that this Tri Sequential technique is a very safe, easy, consistent and easily reproducible maneuver even in the hands of grass root level health worker which not only gives immediate relief to the troubled patients but neither require any assistant nor hospitalization. We limit our study to the reduction technique only as to answer some other questions like surgical verses conservative management or immobilization in external verses internal rotation (as quoted in some studies) [19-26], A large study with longer follow-up is required.

\section{Bibliography}

1. Blake R and Hoffman J. "Emergency department evaluation and treatment of the shoulder and humerus". Emergency Medicine Clinics of North America 17.4 (1999): 859-876.

2. Hill JA. "Epidemiologic perspective on shoulder injuries". Clinics in Sports Medicine 2.2 (1983): 241-246.

3. Westin CD., et al. "Anterior shoulder dislocation: a simple and rapid method for reduction". American Journal of Sports Medicine 23.3 (1995): 369-372.

4. Beattie TF., et al. "A comparison of the Milch and Kocher techniques for acute anterior dislocation of the shoulder". Injury 17.5 (1986): 349-352.

5. Janecki CJ and Shahcheragh GH. "The forward elevation maneuver for reduction of anterior dislocations of the shoulder". Clinical Orthopaedics and Related Research 164 (1982): 177180.

6. Manes HR. "A new method of shoulder reduction in the elderly". Clinical Orthopaedics and Related Research 147 (1980): 200-222. 
7. Kuhn JE. "Treating the initial anterior shoulder dislocation-an evidence-based medicine approach". Sports Medicine and Arthroscopy Review 14.4 (2006): 192-198.

8. Ashton HR and Hassan Z. "Best evidence topic report. Kocher's or Milch's technique for reduction of anterior shoulder dislocations". Emergency Medicine Journal 23.7 (2006): 570571.

9. Milch H. "Treatment of dislocation of the shoulder". Surgery 3.5 (1938): 732-740.

10. Stimson LA. "An easy method of reduction dislocation of the shoulder and hip". Medical Record 57 (1900): 356.

11. Mirick MJ., et al. "External rotation method of shoulder dislocation reduction". Journal of Applied Chemistry and Environment Protection 8.12 (1979): 528-531.

12. Russell JA., et al. "Reduction of acute anterior shoulder dislocations using the Milch technique: a study of ski injuries". Journal of Trauma 21.9 (1981): 802-804.

13. Yuen MC., et al. "An easy method to reduce anterior shoulder dislocation: the Spaso technique". Emergency Medicine Journal 18.5 (2001): 370-372.

14. Baykal B., et al. "Scapular manipulation technique for reduction of traumatic anterior shoulder dislocations: experiences of an academic emergency department". Emergency Medicine Journal 22.5 (2005): 336-338.

15. O'Connor DR., et al. "Painless reduction of acute anterior shoulder dislocations without anesthesia". Orthopedics 29.6 (2006): 528-532.

16. Leidelmeyer R. "Reduced! a shoulder, subtly and painlessly". Emergency Medicine Journal 9 (1977): 233-234.

17. Zahiri CA., et al. "Anterior shoulder dislocation reduction technique-revisited". Orthopedics 20.6 (1997): 515-521.

18. Pradhan RL., et al "Reduction of acute anterior shoulder dislocations: comparing intraarticular lignocaine with intravenous anesthesia". Journal of Nepal Medical Association 45.162 (2006): 223-227.

19. Itoi E., et al. "Position of immobilization after dislocation of the glenohumeral joint. A study with use of magnetic resonance imaging". The Journal of bone and joint surgery American 83.5 (2001): 661-667.
20. Hatrick C., et al. "Should acute anterior dislocation of the shoulder be treated in external rotation". Transactions of the 48th Annual Meeting of the Orthopedic Research Society MJA 179 (2003): 370-371.

21. Itoi E., et al. "A new method of immobilization after traumatic anterior dislocation of the shoulder: a preliminary study". Journal of Shoulder and Elbow Surgery 12.5 (2003): 413-415.

22. Bottoni CR., et al. "A prospective, randomised evaluation of arthroscopic stabilisation versus nonoperative treatment in patients with acute, traumatic, first-time shoulder dislocations". American Journal of Sports Medicine 30.4 (2002): 576-580.

23. Arciero RA., et al. "Arthroscopic Bankart repair versus nonoperative treatment for acute, initial anterior shoulder dislocations". American Journal of Sports Medicine 22.5 (1994): 589594.

24. Wheeler JH., et al. "Arthroscopic versus nonoperative treatment of acute shoulder dislocations in young athletes". Arthroscopy 5.3 (1989): 213-217.

25. Kirkley A., et al. "Prospective randomised clinical trial comparing the effectiveness of immediate arthroscopic stabilisation versus immobilisation and rehabilitation in first time anterior dislocation of the shoulders". Arthroscopy 15.5 (1999): 507-514

26. Te Slaa RS., et al. "A prospective arthroscopic study of acute first-time anterior shoulder dislocation in the young: A fiveyear follow-up study". Journal of Shoulder and Elbow Surgery 12.6 (2003): 529-534.

\section{Volume 2 Issue 7 July 2019}

(C) All rights are reserved by Abhishek Agarwal., et al. 